\title{
Functional interrogation of autoimmune disease genetics using CRISPR/Cas9 technologies and massively parallel reporter assays
}

\author{
James Ding $^{1}$ D $\cdot$ Antonios Frantzeskos $^{1} \mathbb{D} \cdot$ Gisela Orozco $^{1,2} \mathbb{D}$
}

Received: 30 June 2021 / Accepted: 13 August 2021 / Published online: 10 September 2021

(c) The Author(s) 2021

\begin{abstract}
Genetic studies, including genome-wide association studies, have identified many common variants that are associated with autoimmune diseases. Strikingly, in addition to being frequently observed in healthy individuals, a number of these variants are shared across diseases with diverse clinical presentations. This highlights the potential for improved autoimmune disease understanding which could be achieved by characterising the mechanism by which variants lead to increased risk of disease. Of particular interest is the potential for identifying novel drug targets or of repositioning drugs currently used in other diseases. The majority of autoimmune disease variants do not alter coding regions and it is often difficult to generate a plausible hypothetical mechanism by which variants affect disease-relevant genes and pathways. Given the interest in this area, considerable effort has been invested in developing and applying appropriate methodologies. Two of the most important technologies in this space include both low- and high-throughput genomic perturbation using the CRISPR/Cas9 system and massively parallel reporter assays. In this review, we introduce the field of autoimmune disease functional genomics and use numerous examples to demonstrate the recent and potential future impact of these technologies.
\end{abstract}

Keywords Autoimmune disease $\cdot$ CRISPR/Cas9 $\cdot$ MPRA $\cdot$ Functional genetics

\section{Introduction-the case for functional interrogation}

Autoimmune diseases are diverse and numerous, including rare monogenic disorders such as Aicardi-Goutières syndrome and highly prevalent disorders with a complex genetic contribution to risk, such as rheumatoid arthritis (RA). In the US population, the prevalence of autoimmune diseases is estimated at over $3 \%$ of the population and,

This article is a contribution to the special issue on: Genetics and functional genetics of Autoimmune diseases - Guest Editors: Yukinori Okada \& Kazuhiko Yamamoto

James Ding

james.ding@manchester.ac.uk

1 Centre for Genetics and Genomics Versus Arthritis, Division of Musculoskeletal and Dermatological Sciences, School of Biological Sciences, Faculty of Biology, Medicine and Health, The University of Manchester, AV Hill Building, Oxford Road, Manchester M13 9LJ, UK

2 NIHR Manchester Biomedical Research Centre, Manchester University NHS Foundation Trust, Manchester Academic Health Science Centre, Manchester M13 9WL, UK whilst some individual diseases do not follow these rules, this prevalence is higher amongst women and increases with age [1]. Amongst women, autoimmune diseases are one of the leading causes of death in populations of European ancestry [2,3].

From a pathophysiological perspective, these diseases share a common mechanism, being caused by inappropriate or dysregulated immune responses. However, depending on the disease-specific context, this can lead to an array of different clinical manifestations. When focussed on patient care, it may seem that there is little to be gained from considering these diseases as one group; however, the involvement of common biological pathways presents shared therapeutic targets. For example, whilst first used in the treatment of $\mathrm{RA}$, anti-tumour necrosis factor therapies are used in many autoimmune diseases characterised by chronic inflammation, including Crohn's disease (CD) and inflammatory bowel disease (IBD) [4].

Whilst many therapeutic options have already been identified, these typically are effective only for a proportion of patients, such that there is a need for novel therapies. Genetic studies, identifying mutations or variants associated with disease, can highlight genes or pathways as potential 
therapeutic targets. Drugs supported by genetic evidence are estimated to be twice as likely to proceed through clinical development as those without [5].

The high prevalence of complex genetic autoimmune diseases has enabled population-based genome-wide association (GWA) studies. A series of GWA studies published by the Wellcome Trust Case Control Consortium comprise the foundation of the genetic data available for many autoimmune diseases and have identified hundreds of associated loci [6-15]. These studies have demonstrated that there is a common genetic background predisposing to autoimmunity, with disease-specific genetic associations and environmental factors determining the resulting clinical manifestations [16].

A small number of loci, such as those overlapping the major histocompatibility complex (MHC), carry a disproportionate proportion of heritability and have relatively large effect sizes. Here, the mechanism by which individuals are predisposed to disease can be quite clear. For example, a nonsynonymous, deactivating single nucleotide polymorphism (SNP) in the coding sequence of tyrosine kinase 2 (TYK2) is associated with protection from many autoimmune diseases. As a consequence of this discovery, a TYK2 inhibitor is currently being trialled for use in treating patients with CD, systemic lupus erythematosus (SLE) and psoriasis (Ps), with promising initial results [17].

The majority of loci identified through GWA studies as being associated with autoimmune diseases are found in noncoding regions [18]. What is more, as a result of linkage disequilibrium, the resolution with which an association can be mapped is often limited to a large number of co-inherited SNPs. For these loci, it is often unclear how disease susceptibility is conferred, such that the promise of genetic data leading to therapeutic discoveries has yet to be fully realised. Here, functional genomics has the potential to bridge the gap between genetic data and therapeutic translation, from bench to the bedside.

For a given locus, genetic fine-mapping and functional annotation are often helpful in prioritising a small number of highly credible SNPs that are more likely to be causal (high posterior probability). For many loci, however, it is impossible to resolve associations to a single highly credible SNP or functional element. Furthermore many loci associated with autoimmune disease do not overlap with expression quantitative trait loci (eQTLs) [19], which could implicate a causal gene. For many loci, it is unclear which protein-coding genes are affected by disease-associated variants, let alone in what way, in what context and through what mechanism.

Large collections of epigenomic data from disease-relevant cell types have enabled bioinformatic approaches to link disease-associated variants to putative causal genes. For example, this has been achieved by correlating the accessibility of underlying genetic elements with the transcription of proximal genes [20] or by incorporating chromatin conformation capture data into a model of enhancer activity [21]. Such predictions and the underlying data generate valuable hypotheses for individual loci, which require experimental validation.

In this review, we will discuss methods used to interrogate loci associated with autoimmune disease susceptibility, establishing causal variants, implicated regulatory elements and relevant protein-coding genes. In particular, we will focus on application of CRISPR/Cas9 technologies and massively paralleled reporter assays (MPRAs), highlighting publications where these techniques have been used to great effect in the study of autoimmune diseases or exemplar publications in other diseases. These examples are summarised in Table 1.

\section{Genome editing}

Genome editing has long been used as a means of studying the consequences of disease-associated variants. For example, where a protein-coding gene is strongly implicated, knock-out models have been used to determine the consequences of deficiency in this gene and their potential relevance to disease. Such is the case for protein tyrosine phosphatase, non-receptor type 22 (PTPN22), which is strongly associated with many autoimmune diseases. Mice deficient in PTPN22 have abnormalities in T cell function and development [22]. Similarly, mouse knock-out models have been used to establish a role for TNF alpha-induced protein 3 (TNFAIP3) in systemic autoimmunity [23]. Complete loss of TNFAIP3 in mice led to multi-organ inflammation [24, 25], whilst tissue-specific knockout in dendritic cells, for example, amplified B and T cell activation [25], whilst knockout in macrophages resulted in inflammatory cytokine secretion [24].

As genome editing methods have improved, this experimental approach has become feasible even for non-coding regions. The most significant development in this area has been the discovery of the CRISPR/Cas9 system and its development for genome engineering (reviewed in [26]). In brief, in its canonical form, the CRISPR/Cas9 system consists of a guide RNA (gRNA) targeted endonuclease (Cas9) capable of generating double-strand breaks at desired genomic loci with high efficiency and specificity. These breaks are repaired by the cell's DNA repair pathways and successive rounds of efficient cleavage and repair are only escaped when targeted Cas9 is no longer present or an error occurs during repair, such that the target site is disrupted (Fig. 1).

Experiments performed by Simeonov et al. focussed on rs61839660 represent a relevant example of canonical CRISPR/Cas9-mediated genome editing [27]. This 
non-coding variant is found within an intron of interleukin-2 receptor alpha (IL2RA) and is associated with multiple autoimmune diseases, including type 1 diabetes (T1D) and RA. The local sequence is highly conserved between human and mouse, such that B6 mice are homozygous for the protective allele. Deletion of 12-bp surrounding rs61839660, achieved through CRISPR/Cas9 cleavage and non-homologous end joining (NHEJ), caused a reduction in the number of naïve T-cells that express IL-2RA following 1 day of ex vivo activation. The researchers went on to show that replacing the protective allele with the risk allele, achieved through CRISPR/Cas9 cleavage and homology directed repair (HDR)-mediated repair using an exogenous template, caused the same phenotype that is due to a delay in IL-2RA activation.

Whilst the above example is focussed on transgenic mice, such approaches are also possible in primary human cells. For example, Bourges et al. used multiple guide RNAs to generate deletions ranging from 18 to 50-bp surrounding rs6927172 in primary human T-helper cells [28]. This variant is intergenic, located approximately $200 \mathrm{~kb}$ from TNFAIP3, and is associated with RA, coeliac disease (CeD), IBD and 8 other autoimmune diseases. Upon activation, cells harbouring these deletions had reduced TNFAIP3 expression and showed increased expression of activation marker cluster of differentiation 69 (CD69), as well as increased secretion of cytokines interleukin (IL) 4, IL-17 and interferon gamma (IFN-y).

Whilst much more feasible than with previous technologies, canonical CRISPR/Cas9-mediated genome editing remains labour intensive and low throughput. It is, therefore, generally used only to validate other experimental data or following preliminary experimentation. Such was the case in both of these instances, where preliminary experiments had enabled researchers to focus on a single highly credible SNP. In order to achieve this, higher-throughput methodologies are required and the methods implemented by Simeonov et al., Bourges et al. and others are discussed later in this review.

One approach to increasing the throughput of these experiments is merely to perform them in an arrayed context. Whilst lower throughput than pooled CRISPR screens, this offers more opportunity to assess multiple phenotypes that may be affected by individual gRNAs. For example, in order to map a network of regulatory genes and targets, Freimer et al. used an arrayed approach to knockout (KO) 50 genes found to regulate cytotoxic T-lymphocyte-associated protein 4 (CTLA4), IL2RA and IL-2. The effect of these knockouts was assessed by RNA sequencing (RNA-seq) and assay for transposase accessible elements sequencing (ATAC-seq) [29]. It is hoped that the regulatory network established by Freimer et al. may identify further potential drug targets in addition to CTLA4, IL2RA and IL-2. 
a) Small deletions are the predominant mutagenic consequence of WT Cas9 targeted using a single gRNA

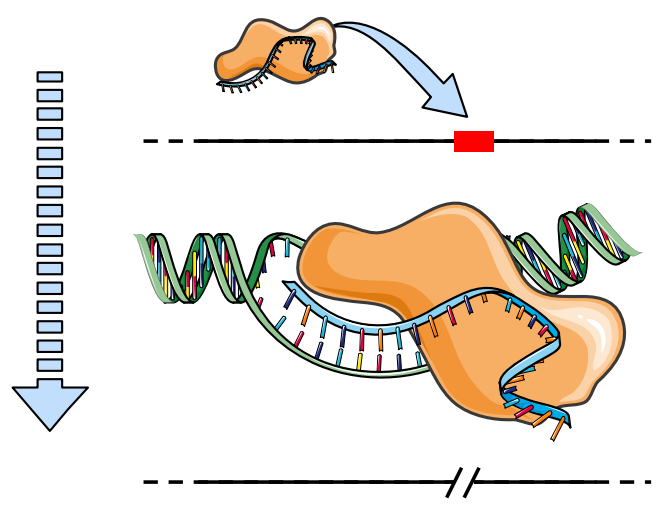

b) dCas9 fusion proteins can be used to activate / repress target chromatin regions c) Both approaches can be scaled up for screens i. GWAS locus

ii. Tiled gRNAs
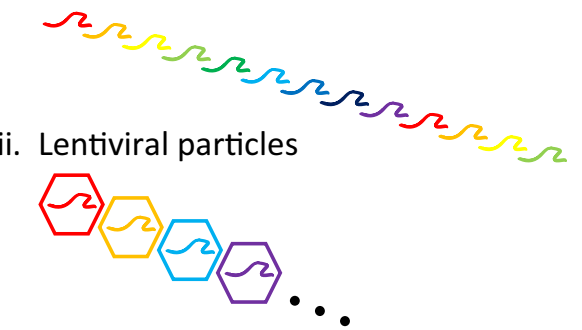

iv. Transduced cells

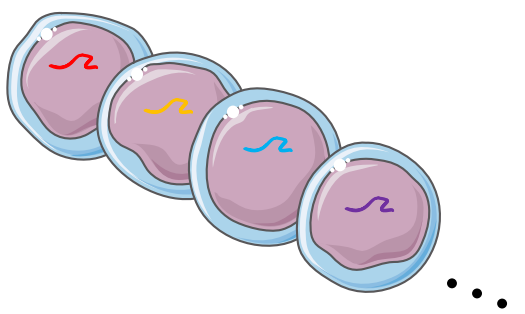

v. scRNAseq/selection and DNA sequencing
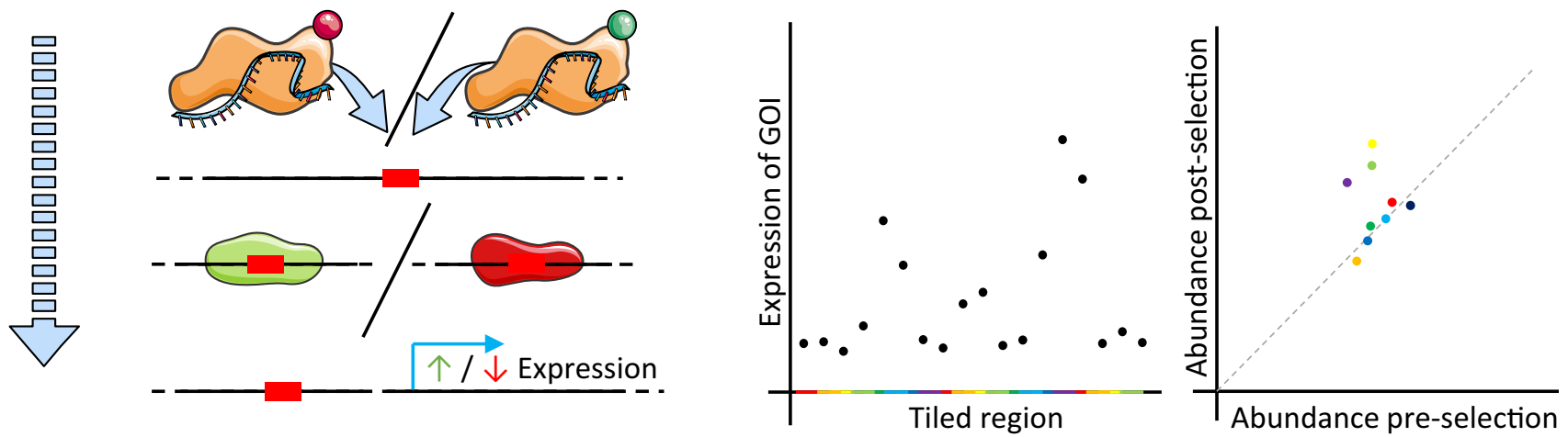

Fig. 1 CRISPR/Cas9-based methods. When targeted to a region of interest (red box) by a single gRNA (blue ribbon), Cas9 (orange) predominately generates small deletions of a few base pairs (a); in coding regions, this is likely to knock out a gene. By fusing chromatin modifiers (red and green circles) to a catalytically inactivated Cas9 protein (dCas9), it is possible to activate or repress regions of interest (b), potentially switching on or off regulatory elements and down-

\section{Massively parallel reporter assays}

MPRAs can be employed to evaluate the regulatory potential of disease-associated loci in a high-throughput manner. These assays use a library of synthesised candidate sequences which are cloned into a reporter vector containing a promoter, reporter and unique barcode. High-throughput sequencing and deconvolution of barcodes are used to measure the influence of candidate regions on transcription, with the prevalence of the barcode in RNA being compared to

stream genes of interest (GOI). In much the same way as for MPRAs, it is possible to tile gRNAs and use lentiviral methods to scale up both of these approaches (c). Screens can be analysed using singlecell RNA sequencing (scRNA-seq), or more classically by measuring the abundance of gRNAs following selection (e.g. drug resistance or cell sorting based on a GOI)

that in DNA [30-32] (Fig. 2). MPRAs can be carried out either in vivo or in vitro (both primary and immortalised cell lines) and are most commonly delivered either by plasmid, or a viral vector (adeno-associated virus (extragenomic) or lentivirus (intragenomic)) [33-35].

MPRAs are commonly applied to explore various regulatory elements, including transcription binding motifs, posttranscriptional regulatory elements and eQTLs. They can be designed to dissect a specific motif or an entire region at a base pair level through multiple perturbations (saturation 
a) GWAS locus

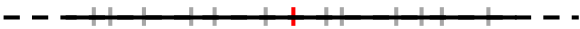

b) Tiled oligos

c) Reporter plasmids

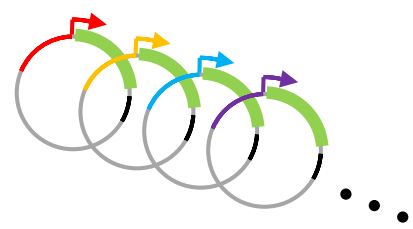

d) Transduced / transfected cells

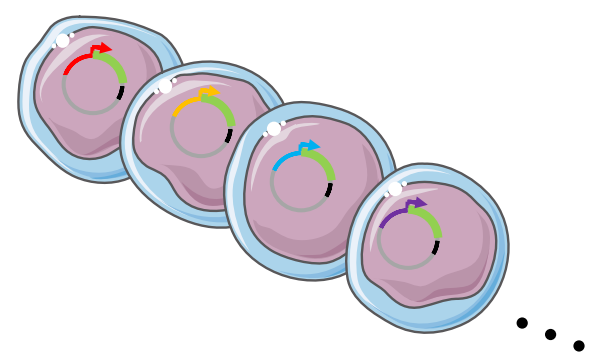

e) High throughput sequencing and data analysis

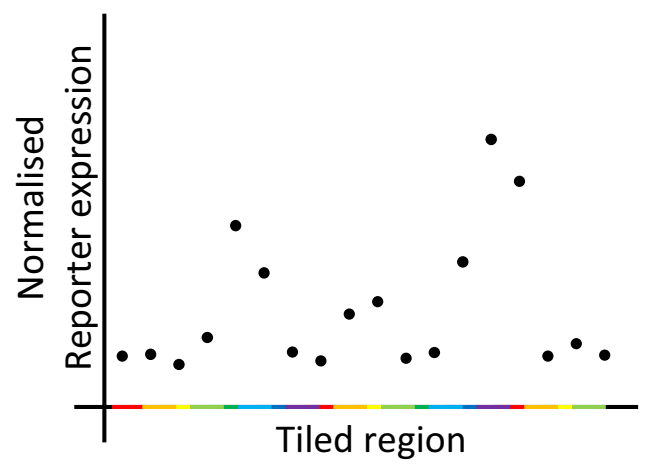

Fig. 2 Experimental overview of massively parallel reporter assays (MPRAs). MPRAs can be targeted to GWAS loci (a) comprised of an index variant (red) and all credible co-inherited variants (grey). Oligos are synthesised across the entire region of interest (b) and cloned into reporter plasmids containing a minimal promoter, reporter gene and unique barcode (c). Plasmids are delivered to a disease-relevant cell type (d) and the effect of the synthesised oligos on reporter gene

mutagenesis) to identify the role (activating/repressing/ none) of each base pair within that region [34, 36, 37]. More commonly when dealing with GWAS variants of unknown function, MPRAs will be designed to compare thousands of

\section{f) Inclusion of disease associated variants}
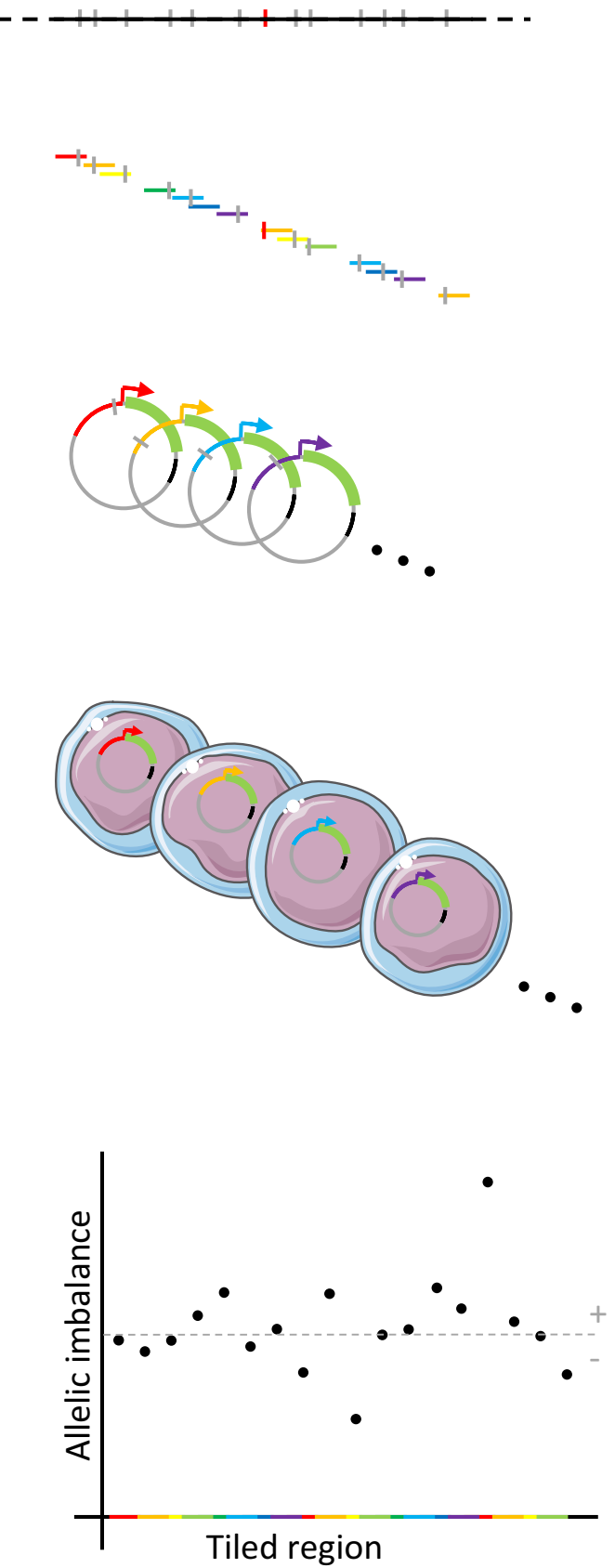

transcription determined. Generally reporter transcripts, quantified by RNA sequencing, are assigned to individual barcodes/oligos and the prevalence of these barcodes/oligos in a DNA-based library is used for normalisation (e). In addition to identifying potential enhancer elements (a-e), MPRAs can be designed to include oligos with both risk and protective alleles for variants of interest, thereby characterising allele-specific enhancer activity (f)

regions containing either protective or risk GWAS SNPs [31, 38]. An example of the scale MPRAs can reach was demonstrated by van Arensbergen et al. who developed a plasmidbased MPRA technology known as survey of regulatory 
elements (SuRE) which aided by high-throughput sequencing can screen millions of SNPs and examine regions up to $2 \mathrm{~kb}$ in length [39].

MPRAs have proved to be a valuable tool for prioritising risk variants that originate from GWA studies. One such study examined 2756 variants in LD with 75 lead SNPs associated to red blood cell traits. A total of 32 variants were found to affect expression in K562 cells, providing a reduced list of candidate variants for functional interrogation in this cell line. Several SNPs were followed up with CRISPR/Cas9-induced deletions. For example, deletion of rs737092 resulted in the modulation of RNA binding motif protein 38 (RBM38) and ribonucleic acid export 1. RBM38 KO demonstrated that this gene played a role in the regulation of alternative splicing in human erythropoiesis [40]. In a similar approach, after establishing the specificity of an MPRA-based strategy by targeting variants in strong LD with eQTLs, Tewhey et al. examined over 9000 variants in 163 GWA loci. The MPRA identified 248 variants with allelic expression differences. An identified functional SNP rs9283753 in LD with a lead SNP for ankylosing spondylitis was edited using a CRISPR/Cas9 HDR approach to produce a homozygous cell line for that variant and confirmed its regulatory role by demonstrating it led to a decrease in the prostaglandin E2 (PTGER4) gene [37].

There are few MPRA studies focusing specifically on autoimmune disease-associated loci, recently Lu et al. screened all 3073 GWAS-linked systemic lupus erythematosus (SLE) variants at 91 loci in lymphoblastoid and T-cell like cell lines: GM12878 and Jurkat. Fifty-one variants showed allele-specific activity in the B cell line and the authors also characterised altered binding of transcription factors (TFs) in particular nuclear factor kappa-light-chainenhancer of activated B cells $(N F \kappa B)$-related TFs. Moreover 92 allele-specific enhancer variants were identified in the $\mathrm{T}$ cell line, $25 \%$ of which were shared with the B cell line [41]. A follow-up study focussed on SNP rs2431697, identified in the aforementioned MPRA, used CRISPR-induced HDR to produce clones homozygous for the reference allele and the risk variant. The risk variant showed a decrease of $\mathrm{NFKB}$ binding and resulted in miR-146a being downregulated, something that has been observed in SLE patients [42].

One study recently applied MPRA in primary T-helper cells for the first time in order to focus on immune-diseaserelated loci, given the enrichment of autoimmune diseaselinked SNPs in T-helper cell regulatory regions [28]. This strategy was successful in validating causality to a single SNP in loci that were well fine-mapped. For example, an IBD locus fine mapped to a single SNP rs 1736137 [43] led to allelic-specific expression differences in both stimulated and unstimulated cells. The same study demonstrated that out of three SNPs with a sum total posterior probability of 99\% in AS (ankylosing spondylitis) only one of the three was found to affect expression. Furthermore, in regions with a greater number of candidate SNPs, the MPRA was capable of prioritising a single SNP in an IBD and MSlinked locus out of 44 candidate SNPs and of discovering a synergistic effect between two SNPs in a T1D locus that showed a greater effect when together as opposed to individually. Bourges et al. examined the TNFAIP3 locus which has been linked with multiple autoimmune diseases including RA, SLE and IBD. In this study, they focused on the fine-mapped IBD-related SNPs [43] and showed rs6927172 was the primary candidate resulting in reduced expression of TNFAIP3 and also reduced NFKB binding, which has previously been associated with super-enhancer formation [44].

When studying MPRA results, it is important to consider some of the limitations this type of reporter assay has. MPRAs generally utilise plasmid DNA which does not consider the native architecture of chromatin; chromatin interactions will not be recapitulated, nor will nearby TF motifs [45]. Lentiviral MPRA methods have been developed in order to introduce the plasmid into the genome, which saw more reproducible results than the plasmid-based method [46]. However, lentiviral-based MPRAs still do not reproduce the endogenous genomic context of the studied SNPs [33]. Moreover, most of the aforementioned studies only study a limited number of cell lines, again potentially missing the disease-relevant context in which a SNP plays a functional role. MPRAs alone cannot determine the causality of a SNP and cannot link the SNP to a gene; they are, therefore, best used as a primary screen to reduce the number of candidate SNPs.

Kreimer et al. meta-analysis of MPRAs focused on regions overlapping various observed regulatory elements and identified chromatin accessibility as the best predictive marker for an enhancer and histone 3 lysine 27 acetylation (H3K27ac) as the best predictive chromatin mark [47]. This matches well with our understanding of enhancers based on other techniques and demonstrates the relevance of data originating from MPRAs. However, an unbiased approach by Kheradpour et al. (i.e. observed regulatory marks were not preselected for) showed that even regions where no regulatory mark was apparent could lead to a difference to promoter activity [31]. It is therefore important to consider that MPRAs may generate false positives (low specificity), as the influence of candidate regions is assessed in a highly artificial context. An alternative interpretation could moot that MPRAs offer a very high level of sensitivity; Benton et al. observed eleven different enhancer predicting annotations (including DNase I hypersensitive sites, H3K27ac, $\mathrm{H} 3 \mathrm{~K} 4 \mathrm{Me} 1$ and $\mathrm{p} 300$ binding sites) in four different cell types and found that a significant amount of functional enhancers are overlooked when focusing exclusively on enhancer predictive annotations [48]. 


\section{CRISPR screens}

Pooled CRISPR screens are typically carried out by introducing a large number of gRNAs into cells using viral methods, with a low multiplicity of infection, such that each cell receives only a single guide. The identity of this guide is determined by sequencing following selection for a phenotype of interest, or when generating transcriptomic information from single cells [49]. Wild-type Cas9 is either delivered transiently in addition to the gRNA, or an engineered cell line capable of constitutive/inducible Cas 9 expression is used. In this instance, gRNA targeted Cas9 will predominantly generate small deletions of several nucleotides around their targeted cleavage site. Alternatively, a catalytically inactivated Cas9 molecule (dCas9) fused to various effector molecules can be used, for example VP64 to activate (CRISPRa), or KRAB to repress (CRISPRi) chromatin at the target site [50]. These technologies enable large-scale genetic screening of regulatory elements and GWAS-associated non-coding variants in both cell lines and primary cells [51].

CRISPR screens, similarly to MPRAs, have been utilised to provide information regarding non-coding functional elements. This is achieved either by targeting specific elements of interest or by tiling entire regions of interest with gRNAs in an unbiased approach [52-56]. Canver et al. were one of the first groups to attempt tiling gRNAs over non-coding DNAse hypersensitivity sites (DHSs) in a pooled screen aiming to identify intronic B-cell lymphoma/leukaemia 11A (BCLL11A) enhancers in the HUDEP-2 (human erythroid progenitor) cell line. Foetal haemoglobin $(\mathrm{HbF})$ was used as an output phenotype as it is known that $B C L 11 A$ reduction leads to an increase in $\mathrm{HbF}$. In contrast to MPRAs, this methodology ensured that, within reason, the genetic and epigenetic context of the underlying sequence was maintained in addition to ensuring that any readout was related to a specific gene and pathway. In one of the DHSs, they found a 42-bp region targeted by 10 different gRNAs that resulted in the most significant change in HbF [57]. Further direct comparison of MPRAs and CRISPR screens is provided in Table 2.

Simeonov et al. applied CRISPRa screening to interrogate autoimmune disease susceptibility loci associated with CD69 and IL2RA. Tiling CRISPR/dCas9-VP64 in Jurkats over the risk region and sorting cells based on expression of either CD69 or IL2RA led to the identification of potential regulatory elements which they referred to as CRISPRaresponsive elements (CaRE). Three such sites were identified in the CD69 locus and 6 in the IL2RA locus, one of which contains the autoimmunity risk variant rs61839660. It was this CaRE that formed the focus of their genome editing work in B6 mice that has already been described [27].

Fulco et al. utilised the complementary CRISPRi method to examine the autoimmunity-linked MYC locus and GATAI region employing 98,000 gRNAs which successfully detected enhancers for those genes [52]. The same group coupled CRISPR/dCas9-KRAB with RNA fluorescence in situ hybridization (FISH) and flow cytometry (CRISPRiFlowFISH), fluorescently labelling candidate gene transcripts in gRNA transduced cells that are then sorted based on expression levels. CRISPRi-FlowFISH was used to target all DHSs near selected genes of interest and measure their expression, revealing single regulatory elements regulating multiple genes and conversely an individual gene being regulated by several enhancers in K562 cells. The group

Table 2 Summary of key attributes of MPRAs and CRISPR screens. Additional details, such as throughput, cost and time, are omitted as they are widely variable and largely overlapping, depending largely on the precise experimental design

\begin{tabular}{|c|c|c|}
\hline & MPRAs & CRISPR screens \\
\hline Methodology & $\begin{array}{l}\text { Fragments of interest inserted in reporter plasmid, with the } \\
\text { effect of individual fragments on expression inferred from } \\
\text { their abundance in reporter transcripts }\end{array}$ & $\begin{array}{l}\text { WT or modified Cas } 9 \text { targeted to genomic loci of interest using } \\
\text { gRNAs, with the impact of individual gRNAs on a specific } \\
\text { phenotype inferred by their abundance following selection for } \\
\text { that phenotype. }\end{array}$ \\
\hline Strengths & - Potential to directly infer allele-specific effects & $\begin{array}{l}\text { - Retention of genomic context } \\
\text { - Any selectable phenotype can be used in addition to single- } \\
\text { cell approaches }\end{array}$ \\
\hline Limitations & $\begin{array}{l}\text { - The size of fragments of interest that can be inserted is } \\
\text { limited } \\
\text { - Susceptible to false positives in enhancer discovery, as the } \\
\text { genomic context of individual fragments is lost } \\
\text { - Reporter plasmid design requires optimisation for different } \\
\text { cell types }\end{array}$ & $\begin{array}{l}\text { - Limited resolution given that gRNAs can be designed to a } \\
\text { limited proportion of the genome } \\
\text { - Susceptible to false negatives as gRNAs have variable efficacy } \\
\text { - The delivery of components, especially modified Cas9 mol- } \\
\text { ecules to many cell types is limiting }\end{array}$ \\
\hline
\end{tabular}

${ }^{a}$ In single-cell RNA sequencing-based CRISPR screens, the transcriptional profile of cells with individual gRNAs is measured. ${ }^{b}$ Delivery of reporter plasmids is also limiting, but to a lesser extent 
subsequently developed an activity-by-contact $(\mathrm{ABC})$ model that combined DHS and H3K27ac occupancy with chromosomal interaction data to predict enhancer-gene links [58]. The ABC model was used to develop enhancer-gene connections in 74 different cell types. ABC enhancers were enriched for GWAS variants and the model was able to link IBD credible SNPs to known IBD-linked genes like IL10, as well as linking new genes such as peptidyl-prolyl cis-trans isomerase (to SNP rs1250566). Novel links generated using their model were validated with CRISPRi-FlowFISH [21].

The aforementioned CRISPRa/i screens rely on a cell line constitutively expressing dCas9. Previous studies in primary cells focused on transgenic mice expressing Cas 9 or dCas 9 for screening murine immune cells or used CRISPR/Cas9 ribonucleoproteins (RNP) to drive HDR or NHEJ in primary human cells. Both murine and human primary immune cell gene KO screens have been helpful in investigating immune-related circuitries [51, 59-65]. A primary murine T cell CRISPR KO screen, performed by Henriksson et al., examined genes involved in the activation and differentiation of murine T-helper type 2 cells (Th2). In combination with ATAC-seq and ChIP-seq for key TFs, the screen showed a significant overlap in genes regulating differentiation and activation and revealed known genes like signal transducer and activator of transcription 6 (Stat6) as well as identifying new Th2 regulating genes [65]. Whilst another group optimised CRISPR RNP delivery in murine innate immune cell types [66], Shifrut et al. developed single guide RNA lentiviral infection with Cas9 protein electroporation (SLICE) to employ a genome-wide CRISPR screen in human primary cytotoxic T cells utilising 77,441 gRNAs targeting 19,114 genes to identify genes with a role in proliferation following stimulation [51]. Selected hits from the screen were examined in-depth by CRISPR perturbation this time combined with single-cell RNA sequencing in both stimulated and unstimulated cells. The same technique was used in a recent study that took a different approach, initially inspecting upstream regulators of 3 genes vital for immune-related pathways, CTLA4, IL2RA and $I L-2$. This was followed by $\mathrm{KO}$ of 24 identified upstream regulators to investigate the downstream gene networks [29]. Similar CRISPR-based perturbation assays have also been applied in primary T-regulatory (Treg) cells targeting selected TFs in various cytokine conditions to reveal regulators of forkhead box P3, CTLA-4 and $I F N-\gamma$ to help understand Treg states [64]. Only recently has delivery of dCas 9 fusion proteins been accomplished in primary $\mathrm{T}$ cells (with the dCas9 protein being delivered using a lentiviral vector) [67]. At the time of writing, the study is yet to be peer reviewed. The authors use both CRISPRa and CRISPRi to map $I L-2$ and $I F N-\gamma$ gene networks.

CRISPR methods come with some limitations. Cas9 binding is restricted by its PAM site, making it challenging to target all candidate sites [68], especially complex, repetitive regions such as the major histocompatibility complex. Highly repetitive regions are also less amenable to MPRAs, but to a lesser extent. Moreover, CRISPRbased techniques are associated with potential off-target effects that need to be controlled for, for example by validating results with additional gRNAs [69]. CRISPR-associated proteins from other bacterial species with different PAM sites have been developed, in addition to engineering of the widely used Streptococcus pyogenes Cas9, in order to increase the percentage of the genome that can be targeted, improve efficiency and reduce off-target effects. CRISPR screens unlike MPRAs cannot currently be utilised for saturation mutagenesis; however, this may be possible with newly developed technologies such as prime [70] and base editing [71]. Base editors (BEs) utilise Cas9nickase, which only cuts a single strand, or dCas 9 fused to a deaminase. Currently two types of BEs exist, cytosine and adenine base editors which can convert $\mathrm{C} \rightarrow \mathrm{T}$ or $A \rightarrow G$, respectively [71]. Prime editing also makes use of Cas9-nickase but is fused to a reverse transcriptase which uses the specifically designed prime editing gRNA, which contains the desired edit, as a template to be copied into the genome. Both these new technologies improve on single base substitution efficiency by overcoming the need for HDR-dependant repair following DSBs [70]. Whilst not yet used in the context of autoimmune diseases, BEs have recently been applied in a screen for the first time targeting the $B R C A l$ coding region to determine the function of variants of unknown significance [72]. Prime editing is a more recent technology and has not been developed for high-throughput assays yet; however, it is likely to be a dominant tool in future functional studies.

The strength of screens and CRISPRi in particular to prioritise disease-related non-coding variants was demonstrated by Ray et al. They compared seven assays including perturbational assays: CRISPRa-FlowFISH and CRISPRiFlowFISH; observational assays: DNase I-seq, ATAC-seq and ChIP-seq (observing H3K27ac); and both lentiviral and transfection-based MPRAs targeting all common variants at the autoimmune-associated TNFAIP3 locus, determining to what extent each assay enriches for disease-linked SNPs and thus how well the assay could prioritise causal SNPs [46]. Using all of these methods in T-cell, B-cell and monocyte cell lines, they found that GWAS SNPs were only enriched amongst accessible chromatin (ATAC-seq peaks) and regions of chromatin that when targeted by CRISPRi lead to a change in TNFAIP3 expression. It is important to note that CRISPRi gRNAs were only designed to regions of accessible chromatin and that not all variants determined to be regulatory overlapped between assays. This emphasises the variability of assays in different contexts, the necessity to combine multiple assays in different cell lines to prioritise 
disease-linked variants and the benefit of combining both observational and perturbation methods.

\section{Conclusions}

Understanding the genetic mechanisms underlying autoimmune diseases is still a major challenge. Appropriately characterising GWAS SNPs in different cell types and contexts can assist in defining relevant genes and pathways. Advances in screening techniques of high-throughput perturbation and genome editing have allowed examination of non-coding autoimmune-associated SNPs and immunerelated pathways at scale, prioritising certain SNPs that can then be studied thoroughly, including at the single base pair level in order to establish causative variants and genes.

Integrating epigenetic data with risk SNPs has helped to identify relative cell types, contexts that variants are likely to act in and potential target genes [73, 74]. The EpiMap project, for example, correlated epigenomic marks and gene expression data from a large number of samples in order to predict tissue-specific links between enhancer and genes [20].

To date, variant interrogation has been predominantly carried out in cell lines. Recent efforts by various groups have optimised the same techniques used in cell lines in some of the more relevant primary human and murine immune cell types; however, a greater range of primary cell types is likely required in order to accurately model all autoimmune disease associations.

Functional screens have helped identify causal variants and improved our understanding of immune networks. Whilst novel autoimmune therapies are yet to arise as a direct consequence of CRISPR or MPRA-based screens, CRISPR screens in cancer have demonstrated their utility in identifying therapeutic targets. For example, a genomewide loss-of-function screen examining the $\beta$-catenin signalling pathway identified KMT2A as a potential target and in vitro validation confirmed that two KMT2A-menin suppress $\beta$-catenin-active colorectal cancer cells [75].

Both CRISPR screens and base pair editing methods will benefit from the current focus and rapid development of CRISPR technology. Whilst they have not attracted the same level of focus, it is clear that MPRA methods also have great potential for improvement, including extending the range of disease-relevant primary cell types used, improving the reporter plasmids used and increasing the size of region that can be incorporated.

Currently a minority of GWAS autoimmune SNPs have been fully characterised. Just as there have been major advances in the availability of epigenetic data for generating testable hypotheses and in the tools available in order to test these in a low-throughput manner, MPRAs and CRISPR screens have emerged as informative and tractable approaches to interrogating autoimmune disease genetics. Further application of these methods, along with incremental improvement, and careful curation and integration of the resulting data will undoubtedly lead to an improved understanding of autoimmune diseases.

Acknowledgements Figures were created through adaptation of Servier Medical Art images (https://smart.servier.com/), produced by Servier and licensed under a Creative Commons Attribution 3.0 Unported License (CC BY 3.0), allowing copying, adaptation and redistribution of content, with attribution (https://creativecommons.org/licenses/by/3.0/).

Funding This work was supported by the Wellcome Trust (award reference 207491/Z/17/Z), Versus Arthritis (award reference 21754) and the NIHR Manchester BRC.

Data availability Not applicable.

Code availability Not applicable.

\section{Declarations}

Conflict of interest The authors declare no competing interests.

Open Access This article is licensed under a Creative Commons Attribution 4.0 International License, which permits use, sharing, adaptation, distribution and reproduction in any medium or format, as long as you give appropriate credit to the original author(s) and the source, provide a link to the Creative Commons licence, and indicate if changes were made. The images or other third party material in this article are included in the article's Creative Commons licence, unless indicated otherwise in a credit line to the material. If material is not included in the article's Creative Commons licence and your intended use is not permitted by statutory regulation or exceeds the permitted use, you will need to obtain permission directly from the copyright holder. To view a copy of this licence, visit http://creativecommons.org/licenses/by/4.0/.

\section{References}

1. Cooper GS, Stroehla BC (2003) The epidemiology of autoimmune diseases. Autoimmun Rev 2:119-125. https://doi.org/10. 1016/S1568-9972(03)00006-5

2. Thomas SL, Griffiths C, Smeeth L et al (2010) Burden of mortality associated with autoimmune diseases among females in the United Kingdom. Am J Public Health 100:2279-2287. https:// doi.org/10.2105/AJPH.2009.180273

3. Walsh SJ, Rau LM (2000) Autoimmune diseases: a leading cause of death among young and middle-aged women in the United States. Am J Public Health 90:1463-1466. https://doi.org/10. 2105/AJPH.90.9.1463

4. Udalova I, Monaco C, Nanchahal J, Feldmann M (2016) Anti-TNF therapy. In: Myeloid cells in health and disease. American Society of Microbiology, pp 637-648. https://doi.org/10.1128/97815 55819194.ch35

5. Nelson MR, Tipney H, Painter JL et al (2015) The support of human genetic evidence for approved drug indications. Nat Genet 47:856-860. https://doi.org/10.1038/ng.3314 
6. Barton A, Thomson W, Ke X et al (2008) Rheumatoid arthritis susceptibility loci at chromosomes $10 \mathrm{p} 15,12 \mathrm{q} 13$ and $22 \mathrm{q} 13$. Nat Genet 40:1156-1159. https://doi.org/10.1038/ng.218

7. Todd JA, Walker NM, Cooper JD et al (2007) Robust associations of four new chromosome regions from genome-wide analyses of type 1 diabetes. Nat Genet 39:857-864. https://doi.org/10.1038/ ng2068

8. Anderson CA, Massey DCO, Barrett JC et al (2009) Investigation of Crohn's disease risk loci in ulcerative colitis further defines their molecular relationship. Gastroenterology 136:523. https:// doi.org/10.1053/j.gastro.2008.10.032

9. Imielinski M, Baldassano RN, Griffiths A et al (2009) Common variants at five new loci associated with early-onset inflammatory bowel disease. Nat Genet 41:1335-1340. https://doi.org/10.1038/ ng. 489

10. Fisher SA, Tremelling M, Anderson CA et al (2008) Genetic determinants of ulcerative colitis include the ECM1 locus and five loci implicated in Crohn's disease. Nat Genet 40:710-712. https://doi.org/10.1038/ng.145

11. Barrett JC, Lee JC, Lees CW et al (2009) Genome-wide association study of ulcerative colitis identifies three new susceptibility loci, including the HNF4A region. Nat Genet 41:1330-1334. https://doi.org/10.1038/ng.483

12. Barrett JC, Hansoul S, Nicolae DL et al (2008) Genome-wide association defines more than 30 distinct susceptibility loci for Crohn's disease. Nat Genet 40:955-962. https://doi.org/10.1038/ng.175

13. Thomson W, Barton A, Ke X et al (2007) Rheumatoid arthritis association at 6q23. Nat Genet 39:1431-1433. https://doi.org/10. 1038/ng.2007.32

14. Nejentsev S, Howson JMM, Walker NM et al (2007) Localization of type 1 diabetes susceptibility to the MHC class I genes HLA-B and HLA-A. Nature 450:887-892. https://doi.org/10.1038/natur e06406

15. Burton PR, Clayton DG, Cardon LR et al (2007) Genome-wide association study of 14,000 cases of seven common diseases and 3,000 shared controls. Nature 447:661-678. https://doi.org/10. 1038/nature05911

16. Ramos PS, Shedlock AM, Langefeld CD (2015) Genetics of autoimmune diseases: insights from population genetics. J Hum Genet 60:657-664. https://doi.org/10.1038/jhg.2015.94

17. Burke JR, Cheng L, Gillooly KM et al (2019) Autoimmune pathways in mice and humans are blocked by pharmacological stabilization of the TYK2 pseudokinase domain. Sci Transl Med 11:1736. https://doi.org/10.1126/scitranslmed.aaw 1736

18. Farh KK-H, Marson A, Zhu J et al (2015) Genetic and epigenetic fine mapping of causal autoimmune disease variants. Nature 518:337-343. https://doi.org/10.1038/nature13835

19. Chun S, Casparino A, Patsopoulos NA et al (2017) Limited statistical evidence for shared genetic effects of eQTLs and autoimmune-disease-associated loci in three major immune-cell types. Nat Genet 49:600-605. https://doi.org/10.1038/ng.3795

20. Boix CA, James BT, Park YP et al (2021) Regulatory genomic circuitry of human disease loci by integrative epigenomics. Nature 590:300-307. https://doi.org/10.1038/s41586-020-03145-z

21. Nasser J, Bergman DT, Fulco CP et al (2021) Genome-wide enhancer maps link risk variants to disease genes. Nature 17:1-6. https://doi.org/10.1038/s41586-021-03446-x

22. Hasegawa K, Martin F, Huang G et al (2004) PEST domainenriched tyrosine phosphatase (PEP) regulation of effector/memory T cells. Science 303:685-689. https://doi.org/10.1126/scien ce. 1092138

23. Lee EG, Boone DL, Chai S et al (2000) Failure to regulate TNFinduced NF- $\mathrm{KB}$ and cell death responses in A20-deficient mice. Science 289:2350-2354. https://doi.org/10.1126/science.289. 5488.2350
24. Matmati M, Jacques P, Maelfait J et al (2011) A20 (TNFAIP3) deficiency in myeloid cells triggers erosive polyarthritis resembling rheumatoid arthritis. Nat Genet 43:908-912. https://doi.org/ $10.1038 / n g .874$

25. Kool M, van Loo G, Waelput $W$ et al (2011) The ubiquitin-editing protein a20 prevents dendritic cell activation, recognition of apoptotic cells, and systemic autoimmunity. Immunity 35:82-96. https://doi.org/10.1016/j.immuni.2011.05.013

26. Hsu PD, Lander ES, Zhang F (2014) Development and applications of CRISPR-Cas9 for genome engineering. Cell 157:12621278. https://doi.org/10.1016/j.cell.2014.05.010

27. Simeonov DR, Gowen BG, Boontanrart M et al (2017) Discovery of stimulation-responsive immune enhancers with CRISPR activation. Nature 549:111-115. https://doi.org/10.1038/nature23875

28. Bourges C, Groff AF, Burren OS, et al (2020) Resolving mechanisms of immune-mediated disease in primary CD $4 \mathrm{~T}$ cells. EMBO Mol Med 12:e12112. https://doi.org/10.15252/emmm. 202012112

29. Freimer JW, Shaked O, Naqvi S, et al (2021) Systematic discovery and perturbation of regulatory genes in human $\mathrm{T}$ cells reveals the architecture of immune networks. bioRxiv 2021.04.18.440363. https://doi.org/10.1101/2021.04.18.440363

30. Melnikov A, Zhang X, Rogov P, et al (2014) Massively parallel reporter assays in cultured mammalian cells. J Visual Exp 51719. https://doi.org/10.3791/51719

31. Kheradpour P, Ernst J, Melnikov A et al (2013) Systematic dissection of regulatory motifs in 2000 predicted human enhancers using a massively parallel reporter assay. Genome Res 23:800-811. https://doi.org/10.1101/gr.144899.112

32. Patwardhan RP, Lee C, Litvin O et al (2009) High-resolution analysis of DNA regulatory elements by synthetic saturation mutagenesis. Nat Biotechnol 27:1173-1175. https://doi.org/10. 1038/nbt. 1589

33. Inoue F, Kircher M, Martin B et al (2017) A systematic comparison reveals substantial differences in chromosomal versus episomal encoding of enhancer activity. Genome Res 27:38-52. https:// doi.org/10.1101/gr.212092.116

34. Ernst J, Melnikov A, Zhang X et al (2016) Genome-scale highresolution mapping of activating and repressive nucleotides in regulatory regions. Nat Biotechnol 34:1180-1190. https://doi.org/ 10.1038/nbt.3678

35. Shen SQ, Myers CA, Hughes AEO et al (2016) Massively parallel cis-regulatory analysis in the mammalian central nervous system. Genome Res 26:238-255. https://doi.org/10.1101/gr.193789.115

36. Movva R, Greenside P, Marinov GK, et al (2019) Deciphering regulatory DNA sequences and noncoding genetic variants using neural network models of massively parallel reporter assays. PLoS ONE 14: https://doi.org/10.1371/journal.pone.0218073

37. Tewhey R, Kotliar D, Park DS et al (2016) Direct identification of hundreds of expression-modulating variants using a multiplexed reporter assay. Cell 165:1519-1529. https://doi.org/10.1016/j.cell. 2016.04.027

38. Melnikov A, Murugan A, Zhang X et al (2012) Systematic dissection and optimization of inducible enhancers in human cells using a massively parallel reporter assay. Nat Biotechnol 30:271-277. https://doi.org/10.1038/nbt.2137

39. van Arensbergen J, Pagie L, FitzPatrick VD et al (2019) Highthroughput identification of human SNPs affecting regulatory element activity. Nat Genet 51:1160-1169. https://doi.org/10.1038/ s41588-019-0455-2

40. Ulirsch JC, Nandakumar SK, Wang L et al (2016) Systematic functional dissection of common genetic variation affecting red blood cell traits. Cell 165:1530-1545. https://doi.org/10.1016/j. cell.2016.04.048 
41. Lu X, Chen X, Forney C et al (2021) Global discovery of lupus genetic risk variant allelic enhancer activity. Nat Commun 12:1611. https://doi.org/10.1038/s41467-021-21854-5

42. Hou G, Harley ITW, Lu X et al (2021) SLE non-coding genetic risk variant determines the epigenetic dysfunction of an immune cell specific enhancer that controls disease-critical microRNA expression. Nat Commun 12:135. https://doi.org/10.1038/ s41467-020-20460-1

43. Huang H, Fang M, Jostins L et al (2017) Fine-mapping inflammatory bowel disease loci to single-variant resolution. Nature 547:173-178. https://doi.org/10.1038/nature22969

44. Brown JD, Lin CY, Duan Q et al (2014) Nf-kb directs dynamic super enhancer formation in inflammation and atherogenesis. Mol Cell 56:219-231. https://doi.org/10.1016/j.molcel.2014.08.024

45. Spicuglia S, Santiago-Algarra D, Dao LTM, et al (2017) Recent advances in high-throughput approaches to dissect enhancer function. F1000Research 6:939

46. Ray JP, de Boer CG, Fulco CP et al (2020) Prioritizing disease and trait causal variants at the TNFAIP3 locus using functional and genomic features. Nat Commun 11:1237. https://doi.org/10. 1038/s41467-020-15022-4

47. Kreimer A, Yan Z, Ahituv N, Yosef N (2019) Meta-analysis of massively parallel reporter assays enables prediction of regulatory function across cell types. Hum Mutat 40:1299-1313. https://doi. org/10.1002/humu.23820

48. Benton ML, Talipineni SC, Kostka D, Capra JA (2019) Genomewide enhancer annotations differ significantly in genomic distribution, evolution, and function. BMC Genomics 20:511. https://doi. org/10.1186/s12864-019-5779-x

49. Shalem O, Sanjana NE, Zhang F (2015) High-throughput functional genomics using CRISPR-Cas9. Nat Rev Genet 16:299-311

50. Horlbeck MA, Gilbert LA, Villalta JE, et al (2016) Compact and highly active next-generation libraries for CRISPR-mediated gene repression and activation. eLife 5:. https://doi.org/10.7554/eLife. 19760

51. Shifrut E, Carnevale J, Tobin V et al (2018) Genome-wide CRISPR screens in primary human T cells reveal key regulators of immune function. Cell 175:1958-1971.e15. https://doi.org/10. 1016/j.cell.2018.10.024

52. Fulco CP, Munschauer M, Anyoha R et al (2016) Systematic mapping of functional enhancer-promoter connections with CRISPR interference. Science 354:769-773. https://doi.org/10.1126/scien ce.aag2445

53. Gasperini M, Hill AJ, McFaline-Figueroa JL et al (2019) A genome-wide framework for mapping gene regulation via cellular genetic screens. Cell 176:377-390.e19. https://doi.org/10. 1016/J.CELL.2018.11.029

54. Sanjana NE, Wright J, Zheng K et al (2016) High-resolution interrogation of functional elements in the noncoding genome. Science 353:1545-1549. https://doi.org/10.1126/science.aaf7613

55. Korkmaz G, Lopes R, Ugalde AP et al (2016) Functional genetic screens for enhancer elements in the human genome using CRISPR-Cas9. Nat Biotechnol 34:192-198. https://doi.org/10. 1038/nbt.3450

56. Borys SM, Younger ST (2020) Identification of functional regulatory elements in the human genome using pooled CRISPR screens. BMC Genomics 21:107. https://doi.org/10.1186/ s12864-020-6497-0

57. Canver MC, Smith EC, Sher F et al (2015) BCL11A enhancer dissection by Cas9-mediated in situ saturating mutagenesis. Nature 527:192-197. https://doi.org/10.1038/nature15521

58. Fulco CP, Nasser J, Jones TR et al (2019) Activity-by-contact model of enhancer-promoter regulation from thousands of CRISPR perturbations. Nat Genet 51:1664-1669. https://doi.org/ 10.1038/s41588-019-0538-0
59. LaFleur MW, Nguyen TH, Coxe MA et al (2019) A CRISPRCas9 delivery system for in vivo screening of genes in the immune system. Nat Commun 10:1669. https://doi.org/10.1038/ s41467-019-09656-2

60. Anderson W, Thorpe J, Long SA, Rawlings DJ (2019) Efficient CRISPR/Cas9 disruption of autoimmune-associated genes reveals key signaling programs in primary human $\mathrm{T}$ cells. J Immunol 203:3166-3178. https://doi.org/10.4049/jimmunol.1900848

61. Parnas O, Jovanovic M, Eisenhaure TM et al (2015) A genomewide CRISPR screen in primary immune cells to dissect regulatory networks. Cell 162:675-686. https://doi.org/10.1016/j.cell. 2015.06.059

62. Freimer JW, Shaked O, Naqvi S, et al Systematic discovery and perturbation of regulatory genes in human $\mathrm{T}$ cells reveals the architecture of immune networks. https://doi.org/10.1101/2021. 04.18 .440363

63. Cortez J, Montauti E, Shifrut E et al (2020) CRISPR screen in regulatory $\mathrm{T}$ cells reveals ubiquitination modulators of Foxp3. Nature 582:416-420. https://doi.org/10.1038/s41586-020-2246-4

64. Schumann K, Raju SS, Lauber M et al (2020) Functional CRISPR dissection of gene networks controlling human regulatory $\mathrm{T}$ cell identity. Nat Immunol 21:1456-1466. https://doi.org/10.1038/ s41590-020-0784-4

65. Henriksson J, Chen X, Gomes T et al (2019) Genome-wide CRISPR screens in T helper cells reveal pervasive crosstalk between activation and differentiation. Cell 176:882-896.e18. https://doi.org/10.1016/j.cell.2018.11.044

66. Riggan L, Hildreth AD, Rolot $\mathrm{M}$ et al (2020) CRISPR-Cas9 ribonucleoprotein-mediated genomic editing in mature primary innate immune cells. Cell Rep 31:107651. https://doi.org/10.1016/j.celrep.2020.107651

67. Schmidt R, Steinhart Z, Layeghi M, et al CRISPR activation and interference screens in primary human $\mathrm{T}$ cells decode cytokine regulation. https://doi.org/10.1101/2021.05.11.443701

68. Westra ER, Semenova E, Datsenko KA, et al (2013) Type I-E CRISPR-Cas systems discriminate target from non-target DNA through base pairing-independent PAM recognition. PLoS Genetics 9: https://doi.org/10.1371/journal.pgen.1003742

69. Zhang XH, Tee LY, Wang XG et al (2015) Off-target effects in CRISPR/Cas9-mediated genome engineering. Molecular Therapy - Nucleic Acids 4:e264. https://doi.org/10.1038/mtna.2015.37

70. Anzalone AV, Randolph PB, Davis JR et al (2019) Searchand-replace genome editing without double-strand breaks or donor DNA. Nature 576:149-157. https://doi.org/10.1038/ s41586-019-1711-4

71. Rees HA, Liu DR (2018) Base editing: precision chemistry on the genome and transcriptome of living cells. Nat Rev Genet 19:770-788. https://doi.org/10.1038/s41576-018-0059-1

72. Kweon J, Jang AH, Shin HR et al (2020) A CRISPR-based baseediting screen for the functional assessment of BRCA1 variants. Oncogene 39:30-35. https://doi.org/10.1038/s41388-019-0968-2

73. Trynka G, Sandor C, Han B et al (2013) Chromatin marks identify critical cell types for fine mapping complex trait variants. Nat Genet 45:124-130. https://doi.org/10.1038/ng.2504

74. Soskic B, Cano-Gamez E, Smyth DJ et al (2019) Chromatin activity at GWAS loci identifies T cell states driving complex immune diseases. Nat Genet 51:1486-1493. https://doi.org/10. 1038/s41588-019-0493-9

75. Wan C, Mahara S, Sun C, et al (2021) Genome-scale CRISPRCas9 screen of $\mathrm{Wnt} / \beta$-catenin signaling identifies therapeutic targets for colorectal cancer. Sci Adv 7: https://doi.org/10.1126/ SCIADV.ABF2567

Publisher's note Springer Nature remains neutral with regard to jurisdictional claims in published maps and institutional affiliations. 